\title{
The MOC31PE immunotoxin reduces cell migration and induces gene expression and cell death in ovarian cancer cells
}

\author{
Merete Thune Wiiger ${ }^{1}$, Hemaseh Bideli ${ }^{1}, \varnothing y s t e i n$ Fodstad ${ }^{1,3}$, Kjersti Flatmark $^{1,2}$ and Yvonne Andersson ${ }^{1 *}$
}

\begin{abstract}
Background: The standard treatment of ovarian cancer with chemotherapy often leads to drug resistance and relapse of the disease, and the need for development of novel therapy alternatives is obvious. The MOC31PE immunotoxin binds to the cell surface antigen EPCAM, which is expressed by the majority of epithelial cancers including ovarian carcinomas, and we studied the cytotoxic effects of MOC31PE in ovarian cancer cells.
\end{abstract}

Methods: Investigation of the effects of MOC31PE treatment on protein synthesis, cell viability, proliferation and gene expression of the ovarian cancer cell lines B76 and HOC7.

Results: MOC31PE treatment for $24 \mathrm{~h}$ caused a dose-dependent reduction of protein synthesis with $\mathrm{ID}_{50}$ values of less than $10 \mathrm{ng} / \mathrm{ml}$, followed by reduced cell viability. In a gene expression array monitoring the expression of 84 key genes in cancer pathways, 13 of the genes were differentially expressed by MOC31PE treatment in comparison to untreated cells. By combining MOC31PE and the immune suppressor cyclosporin A (CsA) the MOC31PE effect on protein synthesis inhibition and cell viability increased tenfold. Cell migration was also reduced, both in the individual MOC31PE and CSA treatment, but even more when combining MOC31PE and CSA. In tumor metastasis PCR arrays, 23 of 84 genes were differentially expressed comparing CSA versus MOC31PE + CSA treatment. Increased expression of the tumor suppressor KISS1 and the nuclear receptor NR4A3 was observed, and the differential candidate gene expression was confirmed in complementary qPCR analyses. For NR4A3 this was not accompanied by increased protein expression. However, a subcellular fractionation assay revealed increased mitochondrial NR4A3 in MOC31PE treated cells, suggesting a role for this protein in MOC31PE-induced apoptotic cell death.

Conclusion: The present study demonstrates that MOC31PE may become a new targeted therapy for ovarian cancer and that the MOC31PE anti-cancer effect is potentiated by CSA.

Keywords: Immunotoxin, EpCAM, Ovarian cancer, Gene-expression, NR4A3, MOC31

\section{Background}

Ovarian cancer is the leading cause of death from gynecological cancers and the patients are commonly diagnosed late with advanced disease. In general, the patients respond well to the primary treatment involving cytoreductive surgery and chemotherapy. However, more than $70 \%$ of the patients relapse, and in the recurrent disease, resistance to chemotherapeutic drugs is common $[1,2]$. New targeted therapies are under evaluation,

\footnotetext{
* Correspondence: yvonne.andersson@rr-research.no

${ }^{1}$ Department of Tumor Biology, Institute for Cancer Research,

Norwegian Radium Hospital, Oslo University Hospital, Oslo, Norway

Full list of author information is available at the end of the article
}

and immunotoxins (ITs) may represent an interesting alternative. ITs consist of an antibody, that with high affinity binds to the target antigen on the cancer cell surface, and a covalently bound toxin. Our MOC31PE immunotoxin binds to the cell surface antigen EpCAM, which is expressed by the majority of epithelial cancers including ovarian carcinomas. Upon internalisation Pseudomonas exotoxin A (PE) inhibits protein synthesis by ADPribosylation of elongation factor 2 and induces apoptosis. EpCAM is a transmembrane glycoprotein, functioning as an epithelial-specific cell-cell adhesion molecule and may be involved in cellular signaling, migration, proliferation, and differentiation [3]. Recently, it has been 
suggested that EpCAM is a cancer stem cell marker and may be expressed by cells undergoing epithelial to mesenchymal transition (EMT), lacking other epithelial markers [4]. EMT-like cellular processes may be important during cancer metastasis, and EpCAM is thus an excellent candidate for therapeutic targeting of epithelial cancers. In a retrospective study of 500 ovarian cancer patients, EpCAM showed consistently high expression across different tumor stages and subtypes [5] and the protein was over-expressed in cancerous tissues compared with non-cancerous ovarian surface epithelium and inclusion cysts [6]. Notably, MOC31PE also induces cell death in chemotherapy-resistant cancer cells [7] and may hence be used in patients with recurrent disease lacking other therapeutic options.

The immune suppressor cyclosporin A (CsA) was introduced in combination with IT to inhibit the host immune response during repeated IT administrations. In parallel with reduced anti-IT antibody production, synergistic cytotoxic effects were observed in vitro and in vivo [8]. The immunosuppressive effect of CsA is caused by binding to cyclophilin A (CypA) [9]. This complex binds and inhibits calcineurin a key enzyme for IL-2 production in T-cells. CypA over-expression has been reported in many human cancers and has also been suggested as a potential therapeutic target [10]. Interestingly, CsA has been reported to reverse chemotherapeutic resistance in patients with recurrent ovarian cancer $[11,12]$. In the present work, we have studied the effects of MOC31PE treatment alone and in combination with CsA on protein synthesis, cell proliferation, viability, and migration on the ovarian cancer cell lines B76 [13] and HOC7 [14], which both express high amounts of EpCAM. Furthermore, MOC31PE-induced alterations in gene transcription were quantified in two different PCR-arrays: Cancer Pathway Finder and Tumor Metastasis.

\section{Materials and methods Materials}

RPMI-1640, PBS, Glutamax, and Hepes were obtained from Lonza (Austria). Fetal calf serum was purchased from PAA (GE Healthcare, UK), MEM w/o leucine, 0.25\% Trypsin/ EDTA from Gibco, and YoYo-1 fluorescent dsDNA staining from Molecular Probes (Life Technologies, UK), and tritiated Leucine from Perkin Elmer (Waltham, MA). Cyclosporine A was purchased from Calbiochem (San Diego, CA) and dissolved in ethanol to $8.3 \mathrm{mM}$ stock solution. The GenElute Mammalian total RNA kit and general laboratory chemicals were from Sigma Aldrich (St. Louis, MO), the Cell Titer 96 AqueousOne solution (MTS) cell proliferation assay was from Promega (Madison, WI). $\mathrm{RT}^{2}$ Profiler PCR Array System, including the cDNA synthesis kit, and SYBR green were from SABiosciences (Qiagen Nordic). Chemicals for validation of gene expression were from Applied (Life Technologies, UK). Plastic ware for cell culture was from Nunc (Thermo Scientific), gels and buffers for protein electrophoresis from Life Technologies, HRP-conjugated antibodies from Dako (DK), and chemiluminescent super-signal substrate from Thermo Scientific.

\section{Cells and immunotoxin}

The human ovarian cancer cell lines B76 [13] and HOC-7 [14] were a gift from Dr C. Marth (Innsbruck Medical University, Innsbruck, Austria). In this study B76 was our main cell line and HOC-7 was used to confirm key results. The cell lines were cultivated in RPMI 1640 medium added Glutamax, Hepes and 8\% heat-inactivated fetal calf serum. The monoclonal antibody MOC31 [15] binds epithelial cell adhesion molecule (EpCAM, CD326) and was conjugated to whole Pseudomonas exotoxin A as previously described [16].

\section{Protein synthesis and cell viability}

The $\left[{ }^{3} \mathrm{H}\right]$-leucine incorporation assay was used to quantify protein synthesis [16] and the Cell Titer 96 AqueousOne solution (MTS) assay was used to determine cell viability as previously described [17].

\section{Cell proliferation, membrane damage and scratch-wound healing in the IncuCyte}

Cells were seeded in 96 well plates and grown to $\approx 50 \%$ confluency, transferred to the IncuCyte (Essen BioSciences, Ann Arbor, Mi) after the medium was replaced with fresh medium with or without IT and/or CsA. Membrane damage was measured after adding YoYo-1, a dye that emit fluorescence when it binds to double-stranded DNA. The cytotoxic index is defined as the number of fluorescent objects in a well, divided by the total number of fluorescent objects obtained after $0.1 \%$ Triton X-100 is added to open all cells in the well. For migration studies, the wound maker tool was used to make scratch wounds in confluent cell culture monolayers in 96 well image-lock plates (Essen BioSciences). Plates were incubated in the IncuCyte for $24 \mathrm{~h}$ and an integrated metric called relative wound density (RWD) was used to quantify effects on migration. This metric measures the cell density in the wound area relative to the cell density outside the wound area.

\section{RNA isolation and PCR array analyses}

The cells were seeded in 6 well plates, grown to $\approx 80 \%$ confluency and treated for $24 \mathrm{~h}$ before RNA was isolated from adherent cells using the GenElute Mammalian total RNA kit (Sigma Aldrich) and quantified in a Picodrop spectrophotometer (Picodrop Ltd, UK). RNA isolated for PCR array assays was treated with DNase I (Invitrogen) and the RNA quality was checked in the UV spectrophotometer. For cDNA synthesis $(1 \mu \mathrm{g} /$ reaction $)$ the $\mathrm{RT}^{2}$ 
first strand kit from SABiosciences was used. The resulting cDNA was diluted and qPCR was run as described in the PCR array protocol (SABiosciences $\mathrm{RT}^{2}$ Profiler PCR Array System) using a BioRad ICycler. Gene expression was tested using either Cancer Pathway Finder (untreated, IT $10 \mathrm{ng} / \mathrm{ml}$ ) - or Tumor Metastasis $(2 \mu \mathrm{M}$ CsA, CsA + IT $10 \mathrm{ng} / \mathrm{ml}$ ) array. There are primers for 84 test genes and 5 reference genes (B2M, HPRT1, RPL13A, GAPDH, and ACTB) on each 96-well plate. Data analysis was performed as described in the protocol from the manufacturer and by using their PCR Array Data Analysis Web portal (www.SABiosciences.com).

\section{Validation of PCR array data}

Gene expression was validated in independent experiments with RNA isolated as described above. The high capacity RNA to DNA master mix was used for cDNA synthesis ( $1 \mu \mathrm{g}$ RNA/ reaction). Gene expression was measured using qPCR analyses with TaqMan probes using the 7500 Real Time PCR machine (Applied Biosystems). Each sample was tested in duplicate. Fold change in expression was calculated using the comparative $C_{t}$ method with RPL37A as a reference gene since the expression of this gene was similar in control and experimental groups. The gene list and corresponding probes are shown in Additional file 1: Table S1.

\section{Subcellular fractionation, gel electrophoresis, and antigen detection}

Cells were grown to $70-80 \%$ confluency in $75 \mathrm{~cm}^{2}$ flasks and treated with MOC31PE and/or CsA for $24 \mathrm{~h}$. The cells were washed with cold PBS and lysed in $500 \mu \mathrm{l} \mathrm{SF}$ buffer (250 mM sucrose, $20 \mathrm{mM}$ Hepes, $10 \mathrm{mM} \mathrm{KCl}$, $1.5 \mathrm{mM} \mathrm{MgCl}_{2}, 1 \mathrm{mM}$ EDTA and $1 \mathrm{mM}$ EGTA, pH 7.5) and the protease inhibitor cocktail was added (MiniComplete, Roche). Cells were scraped from the flasks and the lysates were passed through $25 \mathrm{G}$ needles 10 times, and incubated on ice for $20 \mathrm{~min}$. The nuclear pellet was centrifuged out at $720 \mathrm{~g}$ for $5 \mathrm{~min}$ and the resulting supernatant centrifuged at $10000 \mathrm{~g}$ for $10 \mathrm{~min}$ to separate the cytosolic (supernatant) and mitochondrial (pellet) fractions. Pellets were washed with $500 \mu \mathrm{l} \mathrm{SF}$ buffer, passed through 25G needles 10 times and recentrifuged. Finally, the pellets were resuspended in $50 \mu \mathrm{l}$ lysis buffer (10 mM Tris pH 7.5, 1\% SDS, $1 \mathrm{mM}$ $\mathrm{Na}_{3} \mathrm{VO}_{4}, 0.1 \%$ Triton $\mathrm{X}-100$, and $10 \%$ glycerol) and briefly sonicated. For total cell lysates, cells were lysed in boiling lysis buffer as previously described [17]. Proteins were resolved on 4-12\% Nu-PAGE gels and blotted onto PVDF membranes for antigen detection. The purity of the fractions was validated with antibodies detecting $\alpha$ tubulin (cytosol, Cell Signaling), lamin B1 (nucleus, Abcam), and $\mathrm{F}_{1} \mathrm{~F}_{0}$-ATP synthase (mitochondria, Calbiochem). NR4A3 in the fractions was detected on separate blots using a polyclonal anti-NR4A3/NOR-1 antibody (Novus Biologicals). Chemiluminescence signals were recorded using the G:Box system with a CCD camera from SynGene and quantified using the provided GeneTool software.

\section{Statistical analyses}

Statistical significance was evaluated with two-tailed Students t-test except for qPCR validations where non-parametric Mann-Whitney tests were used. In both tests p-values at 0.05 were considered statistically significant.

\section{Results}

MOC31PE immunotoxin inhibits protein synthesis and reduces cell viability

The ovarian cancer cell line B76 was used to investigate intracellular effects of MOC31PE (IT) and CsA on protein synthesis and cell viability. The expression of EpCAM is high in these cells (as assessed by immunomagnetic selection with MOC31 antibody-coated beads; Additional file 2: Figure $\mathrm{S} 1$ ). The $\mathrm{ID}_{50}$ value for inhibition of protein synthesis was $8 \mathrm{ng} / \mathrm{ml}$ of MOC31PE (Figure 1A). Cell viability was quantified in a MTS assay. In $10 \mathrm{ng} / \mathrm{ml}$ IT-treated cells the viability was decreased to 80 percent of untreated control (Figure 1B). Protein synthesis was inhibited more efficiently when using the combination of IT with $2 \mu \mathrm{M}$ CsA compared to IT treatment alone (Figure 1A). By combining IT with CsA the $\mathrm{ID}_{50}$ value for inhibition of protein synthesis with IT was ten times less than for IT alone. CsA alone showed none or negligible effects on protein synthesis and cytotoxicity. Although $1 \mathrm{ng} / \mathrm{ml}$ IT resulted in 20 percent reduction of protein synthesis, no significant reduction of cell viability was observed after $24 \mathrm{~h}$ (Figure $1 \mathrm{~B}$ ). By extending the incubation period to $48 \mathrm{~h}$, the fraction of metabolically active cells decreased further in all treatment groups (Figure 1B). With $10 \mathrm{ng} / \mathrm{ml}$ IT alone 22 percent cell viability was observed, whereas the addition of CsA reduced the cell survival to only 13 percent.

\section{MOC31PE immunotoxin induces cell membrane damage and reduces cell migration}

Membrane damage was determined by quantifying the number of fluorescent objects in an IncuCyte, where cells were analyzed every second hour for up to $48 \mathrm{~h}$ after adding the fluorescent probe YoYo-1. Addition of YoYo-1 alone did not induce membrane damage. No differences in the number of fluorescent objects were observed during the first $12 \mathrm{~h}$ of treatment, indicating intact cell membranes. The fluorescence increased in IT treated cells after approximately $15 \mathrm{~h}$ (Figure 2A). Figure 2B shows the cytotoxic index (CI) obtained after $48 \mathrm{~h}$ treatment. A dose dependent IT-response was observed with doses from $1 \mathrm{ng} / \mathrm{ml}$ to $100 \mathrm{ng} / \mathrm{ml}$. The membranes of the cells were more damaged by the combination of IT and CsA, 


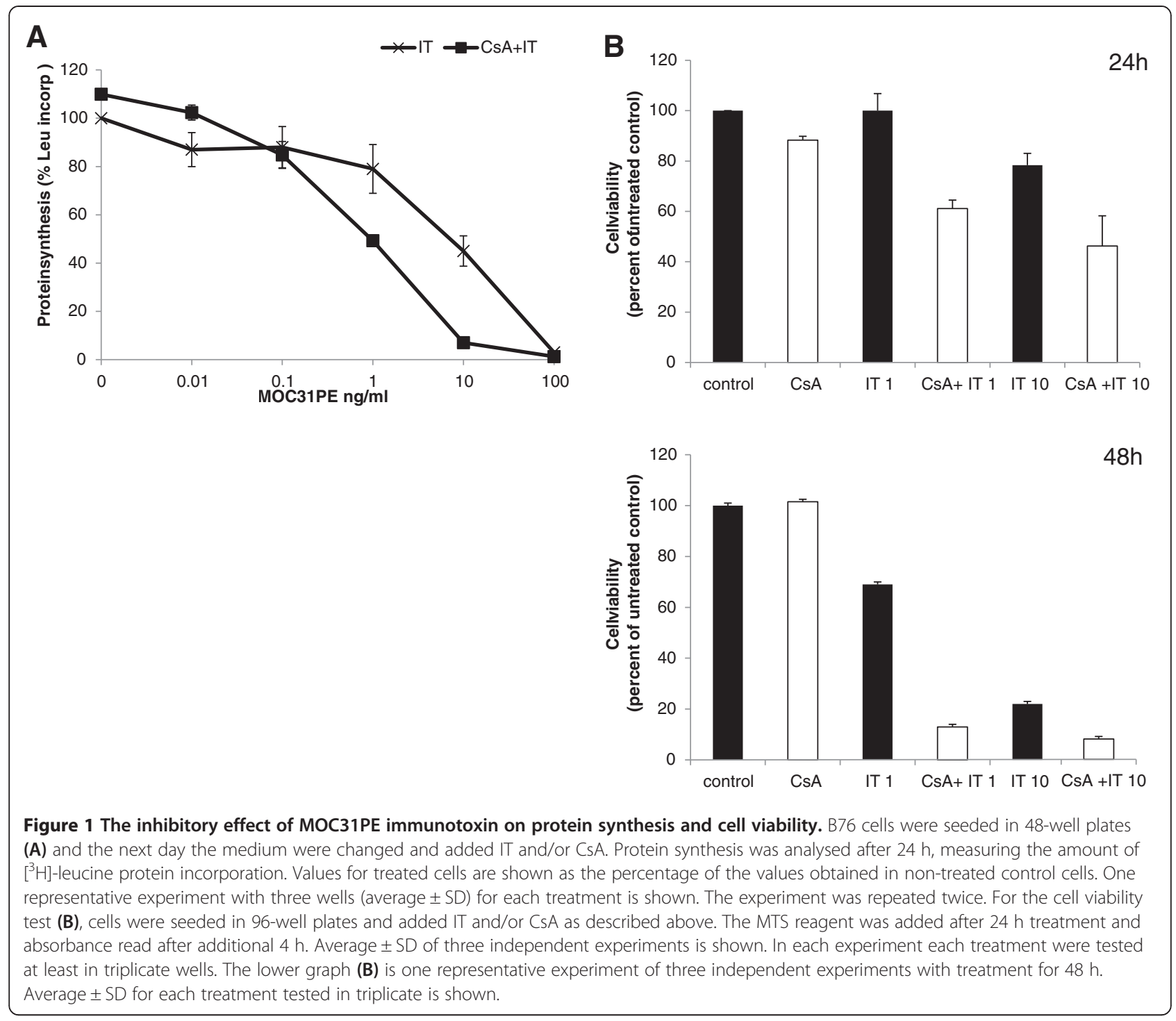

decreasing the IT dose needed by a factor of approximately ten compared to IT alone. Only a minor increase in CI was seen after exposure to CsA alone.

The wound healing assay mimics parts of the cancer metastasis process by measuring in vitro cell migration [18]. In control wells (untreated cells) the relative wound density (RWD) was 91 percent at start of the experiment (average of three wells, Figure 3A) and pictures taken after $22 \mathrm{~h}$ revealed almost complete wound closure (Additional file 3: Figure S2). In wells containing cells treated with IT $(10 \mathrm{ng} / \mathrm{ml})$, cell migration was inhibited as the RWD decreased to 66 percent $(p=0.02)$, and for CsA alone the RWD was 70 percent $(\mathrm{p}=0.005)$. A further reduction was observed when cells were treated with a combination of IT and CsA (RWD $=39$ percent, $\mathrm{p}=0.008$ ). Results from five independent experiments are summarized in Figure 3B.
Effects of MOC31PE immunotoxin on gene expression Previously, microarray analyses have revealed IT-induced differential expression of many transcripts [19]. To focus here on IT-induced changes in gene regulation two different PCR arrays were selected. One aim was to identify which cancer pathways were affected by IT treatment. The tumor metastasis array was used to study effects of the combination of CsA and IT, as this combination was previously shown to increase survival in a metastasis model in nude rats [8]. In two independent experiments, mRNA was isolated from cells treated for $24 \mathrm{~h}$ with $10 \mathrm{ng} / \mathrm{ml}$ IT. Expression of 13 genes was more than twofold changed in IT treated samples compared to nontreated controls (Table 1). Increased gene expression was detected for 11 targets and decreased expression for two targets. The Cq values in the control samples were 25 or more cycles for nine of the 13 affected gene products. Six 


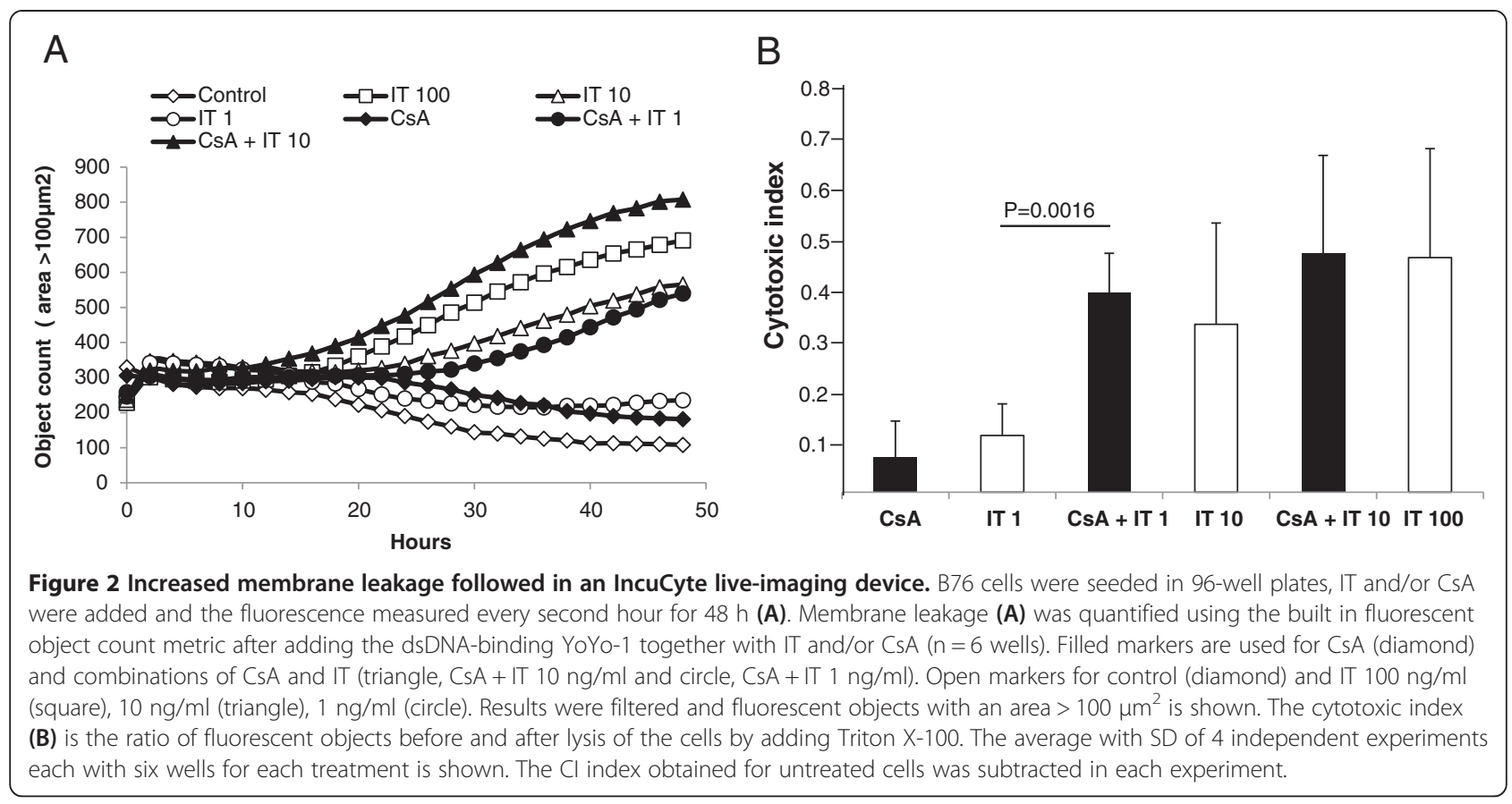

of the detected gene products belong to the angiogenesis pathway. Moreover, increased mRNA levels were found for the transcription factors Jun, ETS2, and NFk $\beta 1$, which e.g. regulate the expression of tumor angiogenesis genes. The highest increase in expression was observed for THBS1 (Thrombospondin, 5.8 fold increase) and PDGF $\beta$ (platelet derived growth factor, 9 fold increase). These genes were selected for validation using qPCR with Taqman probes. RNA was isolated in a set of independent experiments from IT-treated samples and from non-treated controls. In six experiments median fold changed expression for IT treated samples compared to non-treated controls was 5.4 for PDGF $\beta$ (ranging from 2.1 to $31.1, \mathrm{p}<0.02$ ) and 10.5 for THBS1 ( 4.6 to $34.9, \mathrm{p}<0.02)$. The fold change
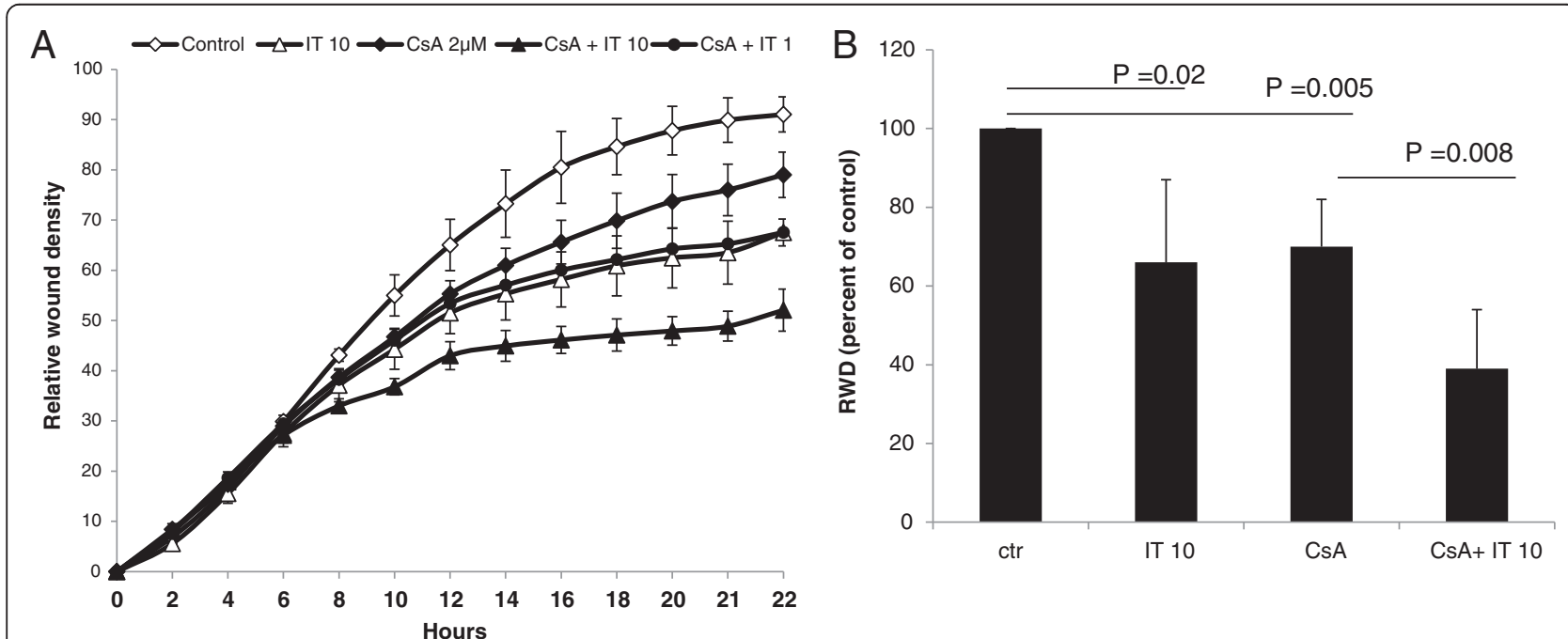

Figure 3 Inhibitory effect of IT +/- CsA in a scratch-wound healing assay followed in an IncuCyte. B76 cells were seeded in 96-well plates (Essen image lock) and scratch wounds made simultaneously in all wells using the wound maker tool. Relative wound density (RWD), defined as the ratio of the cell density in the wound over the cell density outside the wound, was measured every second hour for up to $22 \mathrm{~h}$ (A). Open markers are for control (diamond) and IT $10 \mathrm{ng} / \mathrm{ml}$ (triangle) and filled markers are for CsA (diamond) and the combination of CsA and IT $10 \mathrm{ng} / \mathrm{ml}$ (triangle) or IT $1 \mathrm{ng} / \mathrm{ml}$ (circle). Average RWD after $22 \mathrm{~h}$ in five independent experiments is summarized in (B). The value for the control wells in each experiment is taken as $100 \%$ and used for normalization. Statistical significance was calculated using the T-test (2-tails, unequal variance). 
Table 1 Fold change in gene expression comparing control (untreated cells) and $10 \mathrm{ng} / \mathrm{ml}$ IT treated B76 cells

\begin{tabular}{|c|c|c|c|}
\hline Cancer pathway & Gene & Description & Fold change \\
\hline Adhesion & ITGa3 & Integrin a3 & -2.1 \\
\hline \multirow[t]{6}{*}{ Angiogenesis } & FGFR2 & Fibroblast growth factor receptor 2 & 2.1 \\
\hline & IFNß1 & Interferon $\beta$ & 3.8 \\
\hline & TNF & Tumor necrosis factor & 3.8 \\
\hline & IL8 & Interleukin 8 & 5.0 \\
\hline & THBS1 & Thrombospondin & 5.8 \\
\hline & PDGF $\beta$ & Platelet derived growth factor $\beta$ & 9.0 \\
\hline \multirow[t]{2}{*}{ Cell cycle control and DNA damage repair } & CDC25A & CDC25 phosphatase family & 2.4 \\
\hline & CDKN1A & Cyclin dependent kinase inhibitor & 2.4 \\
\hline \multirow[t]{4}{*}{ Signal transduction molecules and transcription factors } & ERBB2 & Epidermal growth factor receptor family & -2.9 \\
\hline & ETS2 & Transcription factor & 3.2 \\
\hline & NFKß1 & Transcription factor & 3.2 \\
\hline & JUN & Transcription factor & 5.4 \\
\hline
\end{tabular}

Results from two independent experiments are analysed together.

values for the specific mRNA transcript varied between experiments most likely due to high $\mathrm{Cq}$ values i.e. low expression of the mRNA. Within each experiment the variation between technical replicates was low, typically less than 0.5 cycles.

Using qPCR, possible effects of CsA alone and in combination with IT on expression of THBS1 and PDGF $\beta$ were also investigated. In CsA treated cells the expression of THBS1 and PDGF $\beta$ was two-fold reduced $(n=2)$ compared to the expression in untreated control cells. In four independent experiments, the combination treatment compared to CsA alone treatment gave median fold changed expression of 34.5 (from 4.4 to $76.3, \mathrm{p}<0.05$ ) for THBS1 and of 13.9 for PDGF $\beta$ ( 4.5 to $41.3, \mathrm{p}<0.05$ ).

In the Tumor Metastasis Array, 23 of 84 gene products (Table 2) were found to be at least two-fold differentially expressed in the combination treatment compared to CsA alone treatment. Only one mRNA, coding for MYCL-1, was down regulated. The Cq-values for 16 of 23 mRNAs were 25 or higher in CsA-treated cells. Four gene products, coding for NR4A3 (nuclear receptor family 4 member 3), KISS1 (kisspeptin 1), NME4 (expressed in non-metastatic cells 4), and MMP9 (matrix metalloproteinase 9) were selected for validation using $\mathrm{qPCR}$, and the results from the PCR array experiments were confirmed. The median fold changed expression was 16.4 for NR4A3 (ranging from 3 to 25.4, $\mathrm{p}<0.05$ ), and 11.6 for KISS1 (ranging from 3 to $38.5, \mathrm{p}<0.05$ ) in four independent experiments. NME4 was up-regulated 3.8 fold and MMP9 only weakly up-regulated (2.6 times). KISS1 and NR4A3 expression were increased also in cells treated with IT alone, confirming that the differential gene expression was independent of CsA. Expression of these transcripts was also analyzed after IT treatment of the ovarian cancer cell line HOC7. The inhibitory effect of
MOC31PE on protein synthesis and decreased cell viability in HOC7 cells is shown in the Additional file 4: Figure S3 and Additional file 5: Figure S4. The IT induced increase of NR4A3 expression was confirmed in this cell line (Additional file 6: Figure S5) and two-fold increase of THBS1, PDGF $\beta$, and KISS1 transcripts were also detected.

\section{Effects of MOC31PE immunotoxin on NR4A3 protein expression and subcellular localization}

In the tested ovarian cancer cell lines, B76 and HOC7, treated with IT the largest increase in mRNA expression was observed for NR4A3. Immunoblot of B76 protein lysates with anti-NR4A3 antibody gave two proteins bands with apparent molecular weight of 55 and $60 \mathrm{kDa}$. No significant differences in protein level were seen when comparing the different treatments (Figure 4A). Prosurvival as well as pro-apoptotic functions have been ascribed to NR4-family members. The pro-survival effect is due to transcription factor activity and localization in the nucleus, whereas the pro-apoptotic effect has been suggested to require mitochondrial localization. We performed subcellular fractionation to identify the subcellular localization and possibly infer from this the mechanism for NR4A3 involvement during IT treatment. Three fractions enriched in either nuclear proteins, cytosolic proteins, or mitochondrial proteins were obtained and the purity of the different fractions was validated by immunoblotting (Figure 4B). The cytosolic and nuclear fractions were acceptably pure (less than $10 \%$ contamination), whereas the mitochondrial marker protein was detected also in the nuclear fraction, especially when the cells had been treated with IT in combination with CsA. In this case the nuclear fraction contained 24 percent of the total $\mathrm{F}_{1} \mathrm{~F}_{0}-\alpha$ ATP-synthase compared to 4 percent in the corresponding fraction from control cells. 
Table 2 Fold change in gene expression comparing CsA treated B76 cells with or without $10 \mathrm{ng} / \mathrm{ml} \mathrm{IT}$

\begin{tabular}{|c|c|c|c|}
\hline Cancer pathway & Gene & Description & Fold change \\
\hline \multirow[t]{2}{*}{ Cell adhesion } & PNN & Pinin, desmosome-associated protein & 2.2 \\
\hline & FAT1 & Cadherin-related tumor suppressor homolog & 2.3 \\
\hline \multirow[t]{2}{*}{ Cell cyclus } & BRMS1 & Breast cancer metastase suppressor, transcriptional repressor & 2.0 \\
\hline & NF2 & Neurofibromin & 2.2 \\
\hline \multirow[t]{2}{*}{ Cell cyclus and transcription factor } & RB1 & Retinoblastoma, tumor suppressor, transcriptional repressor & 2.2 \\
\hline & TP53 & Tumor suppressor, transcription factor & 2.3 \\
\hline Cell cyclus or cell proliferation & NME1 & Expressed in Non-Metastatic cells, nucleoside diphosphate kinase & 3.2 \\
\hline \multirow[t]{6}{*}{ Cell growth and proliferation } & SSTR2 & Somatostatin receptor 2 , ligand somatostatin $14 / 28$ & 2,1 \\
\hline & DENR & Density-regulated protein, involved in translation & 2.6 \\
\hline & KISS1R & Receptor for KISS1 & 2.8 \\
\hline & FLT4 & Receptor tyrosine kinase, ligand VEGF C/D & 3.1 \\
\hline & CXCR4 & CXC chemokine receptor, ligand SDF-1 & 3.4 \\
\hline & EPHB2 & Receptor tyrosine kinase, ligand ephrin-family members & 10.3 \\
\hline \multirow[t]{2}{*}{ Invasion } & MMP10 & Matrix metalloproteinase & 2.4 \\
\hline & MMP9 & Matrix metalloproteinase & 5.3 \\
\hline \multirow[t]{5}{*}{ Other } & METAP2 & Methionyl aminopeptidase & 2.1 \\
\hline & CD82 & Metastasis suppressor & 2.2 \\
\hline & CTSK & Cathepsin K, cysteine protease & 4.2 \\
\hline & NME4 & Expressed in Non-Metastatic cells, nucleoside diphosphate kinase & 7.6 \\
\hline & KISS1 & Metastasis suppressor & 14.6 \\
\hline \multirow[t]{3}{*}{ Transcription factor } & MYCL1 & Myc-related & -3.8 \\
\hline & SMAD4 & SMAD family member, & 2.0 \\
\hline & NR4A3 & Nuclear-receptor subfamily 4 member $\mathrm{A} 3$, potential transcriptional activator & 20.6 \\
\hline
\end{tabular}

In fractions from control cells NR4A3 was detected mainly as a $60 \mathrm{kDa}$ band in the cytosol and as a $55 \mathrm{kDa}$ band in the mitochondrial fraction. A faint band around $60 \mathrm{kDa}$ was detected in the nuclear fraction, but in this fraction a $100 \mathrm{kDa}$ band was also observed (not shown). IT treatment increased the amount of NR4A3in the mitochondrial fraction, indicating a pro-apoptotic function of NR4A3 (Figure 4B). This increase was also observed in mitochondrial fraction from CsA treated cells, and was accompanied by increased NR4A3 in the nuclear fraction, suggesting increased transcription of NR4A3 regulated genes. NR4A3 was reduced in the mitochondrial fraction from IT + CsA treated cells and further increased in the nuclear fraction. Increased amount of the two NR4A3 bands and detection of the mitochondrial marker in the nuclear fraction indicates altered intracellular compartment for mitochondrial proteins as could be expected in cells undergoing apoptosis.

\section{Discussion}

The major limitation to curative therapy for ovarian cancer is acquired drug resistance to the chemotherapeutic agents used, such as Carboplatin and Paclitaxel. An additional drawback is the induced severe side-effects, mainly caused by the non-cancer cell specificity of the agents, reducing the patients' quality of life. It is therefore necessary to identify novel drugs, which circumvent these disadvantages for successful treatment of ovarian cancer. In the present study, we have demonstrated in several different assays that the MOC31PE effectively inhibits protein synthesis, proliferation and cell survival of ovarian cancer cells, B76 and HOC7. Previously, we have reported in other tumor types synergistic cytotoxic effects of combining MOC31PE and CsA in vitro and in an experimental metastasis model in animals [8]. In agreement with previous results in other tumor types, these effects are potentiated when cells are simultaneously exposed to the immunosuppressant CsA.

The MOC31PE only binds to and kill cells expressing the antigen EpCAM, which is expressed in more than $90 \%$ of all epithelial ovarian carcinomas and to a negligible amount on normal cells, reducing the possibility of IT induced side effects in patients. In a recently conducted Phase I clinical study with MOC31PE, the tolerable profile was satisfactory (Andersson et al., in preparation), which 


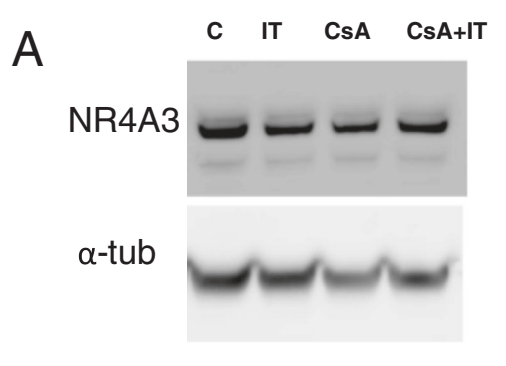

B

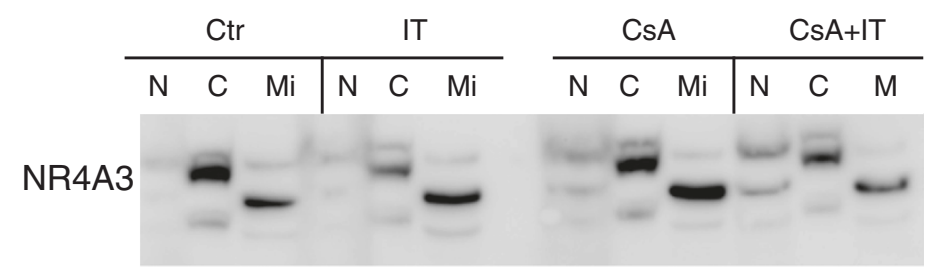

$\operatorname{LaminB}(\mathrm{Nu})$

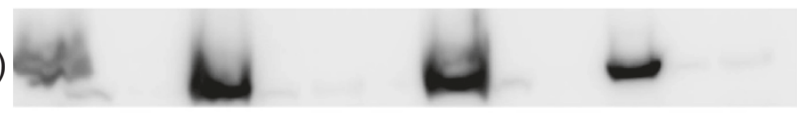

$$
\begin{gathered}
F_{1} F_{0}-\alpha(M i) \\
\alpha \text {-tub (Cyto) }
\end{gathered}
$$

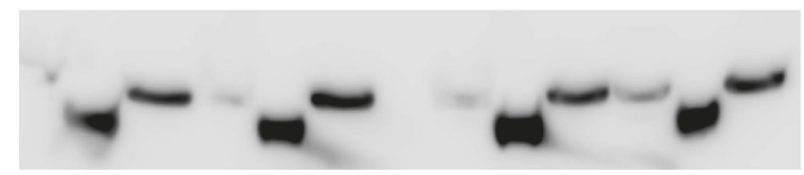

Figure 4 Mechanisms for NR4A3 protein involvement during IT-induced cell death. B76 cells were seeded in $25 \mathrm{~cm}^{2}$ flasks and treated for $24 \mathrm{~h}$ with IT $(10 \mathrm{ng} / \mathrm{ml})$, CsA $(2 \mu \mathrm{M})$ or the combination when cells were $70 \%$ confluent. Cell lysates were prepared and $15 \mu \mathrm{g}$ total protein added each SDS-PAGE lane (A). The apparent molecular weight for the main band is $60 \mathrm{kDa}$ and around $55 \mathrm{kDa}$ for the lower band. In (B) cells were seeded in $75 \mathrm{~cm}^{2}$ flasks and treated as in (A). Adherent cells were used for subcellular fractionation. Immunoblots were probed with the NR4A3 antibody or subcellular fraction marker antibodies as indicated. The immunoblots are from one representative experiment of three independent experiments.

is encouraging for clinical evaluation of MOC31PE against ovarian carcinoma. Interestingly, Phase I and II trials with CsA have shown beneficial effects on chemoresistance in patients with ovarian cancer $[11,20]$ indicating that the combination of MOC31PE and CsA could be used for recurrent ovarian cancer.

Gene expression analysis was performed to identify affected signaling pathways induced by the treatments and several interesting candidate genes were found. In the Cancer Pathway Finder array, the majority of the genes affected by MOC31PE were related to angiogenesis, reflecting the importance of this cancer pathway in B76 cell growth. The two genes with the highest fold increase in expression on the array, PDGF $\beta$ and THBS1, was confirmed by qPCR. The PDGF network was recently identified as a biomarker for prognosis in ovarian cancer where higher levels of PDGF pathway activity were associated with reduced survival [21]. The angiogenesis inhibitor Bevacizumab (Avastin), that binds to VEGF A, is an used molecular target agent in ovarian cancer [22]. Given the importance of the PDGF pathway, targeting of VEGF, PDGF, and FGF at the same time may be more effective than targeting only VEGF [23]. THBS1 was the first endogenous angiogenesis inhibitor identified [24]. A role in cancer progression and cancer inhibition has been ascribed to the protein, and different effects of THBS1 depending on the phase of the progression have been suggested [25]. In an early stage, high stromal expression of THBS1 inhibits tumor growth whereas later in the vascularized tumor THBS1 may increase the adhesive properties of tumor cells or modulate extracellular matrix proteins thereby promoting tumor invasion. We observed that CsA mono-treatment inhibited migration and reduced expression of some transcripts, including THBS1 in addition to potentiating IT effects. Calcineurin, the phosphatase inhibited by CsA, has been reported to regulate transcription of CTSK [26] and CXCR4 [27]; two of five other affected genes. The inhibition of $\mathrm{B} 76$ cell migration by IT + CsA treatment may be a result of reduced THBS1 and/or MMP9 protein levels since increased transcription cannot be accompanied by increased translation due to IT-induced protein synthesis inhibition. In the tumor metastasis array mainly increased gene expression was seen when comparing CsA alone versus 
CsA + MOC31PE treatment of B76 cells. Examples of genes influenced are the metastasis suppressor KISS1 and its receptor. In ovarian carcinoma the increased expression of KISS1 has been shown to inhibit cell migration [28]. This might support the results from the scratch-wound healing assay showing decreased migration in the B76 cells treated with MOC31PE alone or MOC31PE + CsA. Higher expression of KISS1 may also sensitize cancer cells for chemotherapy [29]. Thus our results might support a contribution of MOC31PE as a supplement also to reduce chemotherapy resistance in ovarian cancer treatment.

The largest up-regulation was observed for the nuclear hormone receptor NR4A3, a member of the NR4A subfamily with poorly understood biological function and unknown physiological ligands [30]. Depending on the context, NR4A transcription factors may be pro-survival factors or induce cell death [31]. Knock-out mice without NR4A3 (Nor-1) and NR4A1 (Nur77) developed spontaneous acute myeloid leukemia [32] suggesting tumor suppressing effects. In cancer cells, growth factors and mitogens induce expression of these transcription factors suggesting a role in cancer growth [31]. However, induction of NR4A1 also occurs in response to apoptosis inducing factors in cancer cells. When translocated to mitochondria NR4A1 binds BCL-2, thereby inducing apoptotic cell death [31] and during apoptosis in thymocytes mitochondrial targeting of NR4A3 was observed [33]. In B76 cells, the majority of the NR4A3 protein was located in the cytosol. Two main changes in intracellular distribution were observed. MOC31PE or CsA shifted the protein to the mitochondrial fraction compatible with induction of apoptosis. Especially in MOC31PE + CsA treated cells increased NR4A3 was detected in the nuclear fraction. Increased amount of $60 \mathrm{kDa}$ protein points to increased transcription of its target genes. Since increased $55 \mathrm{kDa}$ protein in the nuclear fraction was accompanied by increased mitochondrial marker protein, and the nuclear fraction was pelleted at low speed, this implies that the mitochondrial mass has increased or that mitochondria have fused to larger structures. This is most likely an effect of the ongoing cell death. The increase in NR4A3 transcript, signals a need for NR4A3 protein synthesis. No corresponding increased NR4A3 protein was detected as IT inhibits protein synthesis, but translocation of NR4A3 to mitochondria enriched fractions suggests a role for this protein in MOC31PE-induced cell-death.

In summary, these results show that a PE-containing IT, MOC31PE, induces transcription of mRNAs for genes involved in angiogenesis and tumor metastasis. In addition, the therapeutic use of MOC31PE alone or in combination with CsA may provide an approach to the treatment of recurrent/chemoresistant ovarian carcinoma, but further investigation is needed to elucidate the effect of MOC31PE and CsA in ovarian cancer models in vivo.

\section{Additional files}

Additional file 1: Table S1. Taqman probe/primers from Applied Biosystems (Life Technology) that were used for validation gene-expression data that were observed with the PCR array technology.

Additional file 2: Figure S1. Cell surface expression of EpCAM was detected using magnetic beads coated with the MOC31 antibody. B76 cells were detached from the plastic with EDTA and incubated for 30 min with these beads or control beads (lgG). Upper panel show very good binding and thus high expression of the antigen EpCAM whereas no binding was seen with control beads.

Additional file 3: Figure S2. Pictures of B76 cells taken immediately after scratching confluent cell layers $(0 \mathrm{~h})$ and after incubating wells with media containing MOC31PE (10 ng/ml) or CSA + MOC31PE for 24 hours in the scratch assay. Control wells were added only growth media. After $24 \mathrm{~h}$ the wound is closed in the control well and still open in treated wells.

Additional file 4: Figure S3. Protein synthesis in $\mathrm{HOC}-7$ ovarian cancer cells after $24 \mathrm{~h}$ incubation with MOC31PE. A dose-dependent decreased incorporation of ${ }^{3} \mathrm{H}$-leu was observed compared with the incorporation of ${ }^{3} \mathrm{H}$-leu in control cells.

Additional file 5: Figure S4. Effect of MOC31PE on HOC-7 ovarian cancer cell viability measured using the MTS-assay. Cells were incubated with IT for 24 and 48 hours as indicated.

Additional file 6: Figure S5. Gene expression of selected genes in HOC-7 ovarian cancer cells tested in QPCR with Taqman probes. RNA was isolated from untreated cells and cells treated with $10 \mathrm{ng} / \mathrm{ml}$ IT in 2-4 independent experiments. Fold-changed expression with standard deviation is shown. The $\mathrm{Cq}$ in control samples were higher than 25 .

\section{Competing interests}

The authors declare that they have no competing financial or non-financial competing interests.

\section{Authors' contributions}

MTW provided, analyzed, and interpreted experimental data and drafted the manuscript. HB was involved in experimental data acquisition, ØF and KF participated in interpretation of the data and revisions of the manuscript, YA substantially contributed to the conception and design of the study, interpretation of experimental data and major revisions of the manuscript. All authors have approved the final version of the manuscript.

\section{Acknowledgments}

We are grateful for the financial support from the Inger and John Fredriksen Foundation for Ovarian Cancer Research. This project has also financially been supported by the Norwegian ExtraFoundation for Health and Rehabilitation through EXTRA funds and the Norwegian Research Council.

\section{Author details}

'Department of Tumor Biology, Institute for Cancer Research,

Norwegian Radium Hospital, Oslo University Hospital, Oslo, Norway.

${ }^{2}$ Department of Gastroenterological Surgery, Norwegian Radium Hospital, Oslo University Hospital, University of Oslo, Oslo, Norway. ${ }^{3}$ University of Oslo, Oslo, Norway.

Received: 25 September 2013 Accepted: 11 February 2014

Published: 15 February 2014

\section{References}

1. Ozols RF, Bundy BN, Greer BE, Fowler JM, Clarke-Pearson D, Burger RA, et al: Phase III trial of carboplatin and paclitaxel compared With cisplatin and paclitaxel in patients with optimally resected stage III ovarian cancer: a gynecologic oncology group study. J Clin Oncol 2003, 21:3194-3200. 
2. Bamias A, Pignata S, Pujade-Lauraine E: Angiogenesis: a promising therapeutic target for ovarian cancer. Crit Rev Oncol/Hematol 2012, 84:314-326.

3. Patriarca C, Macchi RM, Marschner AK, Mellstedt H: Epithelial cell adhesion molecule expression (CD326) in cancer: a short review. Cancer Treat Rev 2011, 38:68-75.

4. Tveito S, Andersen K, Karesen R, Fodstad O: Analysis of EpCAM positive cells isolated from sentinel lymph nodes of breast cancer patients identifies subpopulations of cells with distinct transcription profiles. Breast Cancer Res 2011, 13:R75.

5. Köbel M, Kalloger SE, Boyd N, McKinney S, Mehl E, Palmer C, et al: Ovarian carcinoma subtypes are different diseases: implications for biomarker studies. PLoS Med 2008, 5:1749-1759.

6. Emmanuel C, Gava N, Kennedy C, Balleine RL, Sharma R, Wain G, et al: Comparison of expression profiles in ovarian epithelium In Vivo and ovarian cancer identifies novel candidate genes involved in disease pathogenesis. PLOS ONE 2011, 6:e17617.

7. Risberg K, Fodstad $\varnothing$, Andersson $Y$ : Anti-melanoma activity of the 9.2.27PE immunotoxin in dacarbazine resistant cells. J Immunother 2010, 33:272-278.

8. Andersson Y, Engebraaten O, Fodstad O: Synergistic anti-cancer effects of immunotoxin and cyclosporin in vitro and in vivo. Br J Cancer 2009, 101:1307-1315.

9. Xavier M: Drug immunosuppression therapy for adult heart transplantation. Part 1: immune response to allograft and mechanism of action of immunosuppressants. Ann Thorac Surg 2004, 77:354-362.

10. Obchoei S, Wongkhan S, Wongkham C, Li M, Yao Q, Chen C: Cyclophilin A: potential functions and therapeuti target for human cancer. Med SCi Monit 2009, 15:RA221-RA232.

11. Morgan RJJ, Synold T, Gandara D, Muggia F, Scudder S, Reed E, et al: Phase II trial of carboplatin and infusional cyclosporine with alpha-interferon in recurrent ovarian cancer: a California cancer consortium trial. Int $J$ Gynecol Cancer 2007, 17:373-378.

12. Sood A, Sorosky J, Squatrito R, Skilling J, Anderson B, Buller R: Cyclosporin A reverses chemoresistance in patients with gynecologic malignancies. Neoplasia 1999, 1:118-122.

13. Marth C, Zeimet AG, Herold M, Brumm C, Windbichler G, Müller-Holzner E, et al: Different effects of interferons, interleukin- $1 \beta$ and tumor necrosis factor- $a$ in normal (OSE) and malignant human ovarian epithelial cells. Int J Cancer 1996, 67:826-830.

14. Buick RN, Pullano R, Trent JM: Comparative properties of five human ovarian adenocarcinoma cell lines. Cancer Res 1985, 45:3668-3676.

15. Myklebust AT, Beiske $K$, Pharo A, Davies $C L$, Aamdahl S, Fodstad $\varnothing$ : Selection of anti-SCLC antibodies for diagnosis of bone marrow metastasis. Br J Cancer 1991, 63:49-53.

16. Engebraaten $O$, Sivam G, Juell S, Fodstad $\varnothing$ : Systemic immunotoxin treatment inhibits formation of human breast cancer metastasis and tumor growth in nude rats. Int J Cancer 2000, 88:970-976.

17. Andersson $Y$, Le $H$, Juell $S$, Fodstad $\varnothing$ : AMP-activated protein kinase protects against ant-epidermal growth factor recetor-pseudomonas exotoxin A immunotoxin-induced MA11breast cancer cell death. Mol Cancer Res 2006, 5:1050-1059.

18. Arwert EN, Hoste E, Watt FM: Epithelial stem cells, wound healing and cancer. Nat Rev Cancer 2012, 12:170-180.

19. Risberg K, Guldvik IJ, Palchaudhuri R, Xi Y, Fodstad $\varnothing$, Hergenrother PJ, et al: Triphenylmethyl derivatived enhances the anticancer effect of immunotoxins. J Immunother 2011, 34:438-447.

20. Chambers S, Chambers J, Davis C, Kohorn E, Schwartz P, Lorber M, et al: Pharmacokinetic and phase I trial of intraperitoneal carboplatin and cyclosporine in refractory ovarian cancer patients. J Clin Oncol 1997, 15:1945-1952.

21. Ben-Hamo R, Efroni S: Biomarker robustness reveals the PDGF network as driving disease outcome in ovarian cancer patients in multiple studies. BMC Syst Biol 2012, 6:3

22. Itamochi H: Targeted therapies in epithelial ovarian cancer: molecular mechanism of action. World J Biol Chem 2010, 1:209-2200.

23. Bell-McGuinn K, Konner J, Tew W, Spriggs DR: New drugs for ovarian cancer. Ann Oncol 2011, 22:viii77-viii82.

24. Good DJ, Polverini PJ, Rastinejad F, Le Beau MM, Lemons RS, Frazier WA, et al: A tumor suppressor-dependent inhibitor of angiogenesis is immunologically and functionally indistinguishable from a fragment of thrombospondin. Proc Natl Acad Sci 1990, 87:6624-6628.
25. Kazerounian S, Yee KO, Lawler J: Thrombospondins in cancer. Cell Mol Life Sci 2008, 65:700-712.

26. Combs MD, Braitsch CM, Lange AW, James JF, Yutzey KE: NFATC1 promotes epicardium-derived cell invasion into myocardium. Dev 2011, 138:1747-1757.

27. Cristillo $A D$, Bierer BE: Regulation of CXCR4 expression in human $T$ lymphocytes by calcium and calcineurin. Mol Immunol 2003, 40:539-553.

28. Hata K, Dhar DK, Watanabe $Y$, Nakai H, Hoshiai H: Expression of metastin and a G-protein-coupled receptor (AXOR12) in epithelial ovarian cancer. Eur J Cancer 2007, 43:1452-1459.

29. Jiffar T, Yilmaz T, Lee J, Hanna E, El-Naggar A, Yu D, et al: KiSS1 mediates platinum sensitivity and metastasis suppression in head and neck squamous cell carcinoma. Oncogene 2011, 30:3163-3173.

30. Pearen MA, Muscat GEO: Minireview: nuclear hormone receptor $4 \mathrm{~A}$ signaling: implications for metabolic disease. Mol Endocrinol 2010, 24:1891-1903.

31. Moll U, Marchenko M, Zhang X: p53 and Nurr77/TR3 - transcription factors that directly target mitochondria for cell death induction. Oncogene 2006, 25:4725-4743.

32. Mullican S, Zhang S, Konopleva M, Ruvolo V, Andreeff M, Milbrandt J, et al: Abrogation of nuclear receptors $\mathrm{Nr} 4 \mathrm{a} 3$ and $\mathrm{Nr} 4 \mathrm{a} 1$ leads to development of acute myeloid leukemia. Nat Med 2007, 13:730-735.

33. Thompson J, Burger ML, Whang $\mathrm{H}$, Winoto $\mathrm{A}$ : Protein kinase $\mathrm{C}$ regulates mitochondrial targeting of Nur77 and its family member Nor-1 in thymocytes undergoing apoptosis. Eur J Immunol 2010, 40:2041-2049.

doi:10.1186/1757-2215-7-23

Cite this article as: Wiiger et al:: The MOC31PE immunotoxin reduces cell migration and induces gene expression and cell death in ovarian cancer cells. Journal of Ovarian Research 2014 7:23.

\section{Submit your next manuscript to BioMed Central and take full advantage of:}

- Convenient online submission

- Thorough peer review

- No space constraints or color figure charges

- Immediate publication on acceptance

- Inclusion in PubMed, CAS, Scopus and Google Scholar

- Research which is freely available for redistribution 